\title{
Lattice Boltzmann Computations of the Fluid Flow in the Wake of a Wind Turbine Modelled by a Porous Disk
}

\author{
D. Hamane $e^{1,2 \dagger}$, O. Guerri ${ }^{2}$ and S. Larbi ${ }^{1}$ \\ ${ }^{1}$ Ecole Nationale Polytechnique, El Harrach, Algiers, CP 16200, Algeria \\ ${ }^{2}$ Centre de Développement des Energies Renouvelables, BP 62 Route de l'Observatoire, Bouzareah Algiers, \\ CP 16340, Algeria \\ $\dagger$ Corresponding Author Email: hamanedawoud@gmail.com
}

(Received December 3, 2020; accepted April 4, 2021)

\begin{abstract}
The study of wind turbine wakes was the subject of numerous papers. However, most computational studies were based on Reynolds Averaged Navier Stokes (RANS) equations or on large eddy simulations (LES) technique. In this work, a different technique based on Lattice Boltzmann method (LBM) is applied. This approach allows the numerical investigations of the flow field in the wake of a wind turbine modelled by a solid porous disk. The LBM is a mesoscopic simulation method for fluid flow computations. The applied model is based on the Regularized Bhatnagar-Groos-Krook (R-BGK) model and an LBM-LES method is used to solve the turbulent flow at a Reynolds number $R e=40000$. Three-dimensional computations are performed using the open source Palabos code. The effects of the lattice velocity schemes D3Q19 and D3Q27, the value of Smagorinsky's constant and the type of boundary conditions on the solid porous disk are investigated. The potential of 3D LBM computations to describe the far wake of an horizontal axis wind turbine is also shown.
\end{abstract}

Keywords: LBM; LES; Porous disk; Actuator disk; Wind turbine wake.

\section{NOMENCLATURE}

$\begin{array}{ll}a & \text { induction factor } \\ c & \text { lattice speed of sound } \\ c_{i} & \text { discrete velocities } \\ C_{P} & \text { power coefficient } \\ c_{s} & \text { speed of sound } \\ C_{s} & \text { Smagorinsky constant } \\ C_{T} & \text { thrust coefficient } \\ D & \text { rotor diameter } \\ f_{i} & \text { distribution function } \\ f_{i}^{e q} & \text { equilibrium distribution } \\ f_{i}{ }^{n e q} & \text { functions } \\ k & \text { non-equilibrium distribution } \\ p & \text { functions } \\ Q_{i} & \text { growth rate of the wake } \\ R e & \text { disk porosity } \\ t & \text { Reynolds number } \\ U_{0} & \text { physical time } \\ & \text { free stream velocity }\end{array}$

$\begin{array}{ll}x & \text { streamwise location } \\ y & \text { spanwise location } \\ z & \text { vertical location } \\ z_{h} & \text { hub height } \\ \beta & \text { function of } C_{T} \\ \delta & \text { Kronecker symbol } \\ v & \text { initial wake width } \\ v_{T} & \text { turbulent viscosity } \\ v_{0} & \text { molecular viscosity } \\ v_{e} f f & \text { total viscosity } \\ \omega & \text { constant weight } \\ \Omega & \text { collision operator } \\ \Pi & \text { second order non-equilibium moment } \\ \rho & \text { fluid density } \\ \sigma & \text { standard deviation of the Gaussian-like } \\ \tau_{0} & \text { velocity deficit } \\ \tau_{T} & \text { molecular relaxation time } \\ \tau_{e} f f & \text { turbulent relaxation time } \\ & \text { total relaxation time }\end{array}$




\section{INTRODUCTION}

The use of wind energy for the production of electricity is constantly evolving around the world. This boom is mainly due to a better analysis of the physical phenomena involved in wind energy conversion systems, which has led to a drop in the production costs of electrical energy. Among the most studied subjects, there is that of the wake of wind turbines. Indeed, the wind speed decreases downstream of the wind turbines and the turbulence increases. In the case of wind farms, the wind turbines located downstream are less efficient if the distance separating them from upstream turbines is small. In addition, the turbulence being there more important, the stresses are more important, consequently, the wind turbine blades can be damaged. The study of the wake downstream of the wind turbines has therefore been the subject of numerous studies, both numerical and experimental. Thus, Gomez-Elvira et al. (2005) studied the turbulence characteristics of the wind turbine near wakes. They applied a calculation method based on an explicit algebraic model for the components of the turbulent stress tensor. Kasmi and Masson (2008) combined the Reynolds Averaged Navier Stokes (RANS) equations with the actuator-disk model to compute the flow in the wind turbine wake. Turbulence was modelled by a modified $k-\varepsilon$ model in which an extra term was added to the transport equation for the turbulence energy dissipation rate in the near wake. Ameur et al. (2011) performed 2Daxisymmetric and 3D numerical simulations of flow around two horizontal-axis wind turbines. Their simulations were performed by resolving the RANS equations over the whole computational domain. The rotor was approximated by the actuator disk concept and the geometry of the nacelle was taken into account.

$\mathrm{Wu}$ and Porte-Agel (2011) applied large-eddy simulation (LES) coupled with a wind-turbine model, to characterise the wake of a turbine in a turbulent and neutral boundary-layer flow. Bastankhah and Porte-Agel (2014) introduced an analytical wake model to predict the wind velocity distribution downwind of a wind turbine. Their model was derived by applying conservation of mass and momentum and assuming a Gaussian distribution for the velocity deficit in the wake. Englberger and Dornbrack (2016) used a geophysical flow solver and carried out LES computations to investigate the wake of a wind turbine in a turbulent boundary layer under neutral stratification. In Englberger and Dornbrack (2017), these investigations were performed for a wind turbine under non-neutral thermal stratification. More recently, Ranjbar et al. (2020) performed experimental and numerical investigations on the flow field in the vicinity of porous disks with porosity varying from 0.4 to 0.8 . Relations between the disk porosity and wind turbine parameters including induction factor, power coefficient and thrust coefficient were suggested. These reported works are not exhaustive. More other studies related to this topic were the subject of review papers, among them, Sanderse et al. (2011) and the latest, Porte-Agel et al. (2020).

In short, most of the numerical methods applied for the computations of the fluid flow in the wake of wind turbines were based on the solution of the Reynolds Averaged Navier-Stokes equations (RANS) or on Large Eddy Simulations (LES) in more recent studies. Knowing that LES methods are costly in time and computing equipment, a different numerical technique, namely the Lattice Boltzmann Method (LBM) is applied in this paper, to compute the wind turbine wake. The most advantages of the LBM are its computational efficiency in solving complex physical problems and in some specific areas of CFD (Latt et al. 2020). This is a continuum method and not a diskrete particle approach. It offers an Eulerian view of the flow and is mesh based (Gourdain et al. 2018). Thereby, LB computations were successfully applied for the simulation of natural convection problems with comple.x geometry (Khazaeli et al. 2015), to study the flow and heat transfer of nanofluids (Jafari et al. 2018) and other fluid flow problems in porous media. LBM was also applied for the numerical simulation of viscous flow around bluff body at low Reynolds numbers (Kumar et al. (2010)) and athigh Reynolds numbers (Liu et al. 2008). As for aerodynamic applications, Chen (2015) coupled MRT-LBM with SA turbulent model to compute a two-dimensional flow around NACA0012 airfoil. Gourdain et al. (2018) applied the LBM for the study of the turbulent flow around microair vehicles.

Their results were compared to Direct Numerical Simulations (DNS) of a single blade rotor at low Reynolds number, to LES computations of a twobladed rotor operating inground effect and to LES computations of a three-bladed rotor optimised for acoustic performance. Rullaud et al. (2018) developed a 2D solver based on the LBM coupled to the Actuator Line method to compute the forces acting on vertical axis wind turbines. Their study was applied to two 2D turbine models for the prediction of blade forces and wake velocity. Grondeau et al. (2019) combined also an Actuator Line Method (ALM) with the Lattice Boltzmann Method (LBM) to study vertical axis wind turbines. Their ALM/LBM model was implemented in a LBM/LES solver to compute the wake of a three-bladed H-type Darrieus tidal turbine.

The aim of our investigations is to assess the potential of 3D LB computations to describe the far wake of an horizontal axis wind turbine, modelled by a solid porous disk. This model is similar to the actuator disk concept (Espana et al. (2012)). These simulations are carried out using the open source Palabos code (www.Palabos.org), developed by the University of Geneva. A complete presentation of this code can be found in Latt et al. (2020).

The paper is organized as follows: A description of the considered turbine model is given in section 2 . The applied LBM technique and LES modelling are described in section 3 . Then, the obtained results are discussed in sections 4 . 


\section{WIND TURBINE MODEL}

The porous disk that model the wind turbine is analogous to that described by Aubrun et al. (2013) and Sumner et al. (2013).

These computations are performed for a solid porous disk without mast, of porosity $p=55 \%$ and diameter $D=0.100 m$ (Fig. 1). The reference velocity is $U_{0}=$ $6 \mathrm{~m} / \mathrm{s}$ and the Reynolds number is set to $R e=40000$. These parameters represent a modeled wind turbine with a thrust coefficient $C_{T}=0.42$, a power coefficient $C_{P}=0.37$ and an induction factor $a=$ 0.195 (Espana 2009).

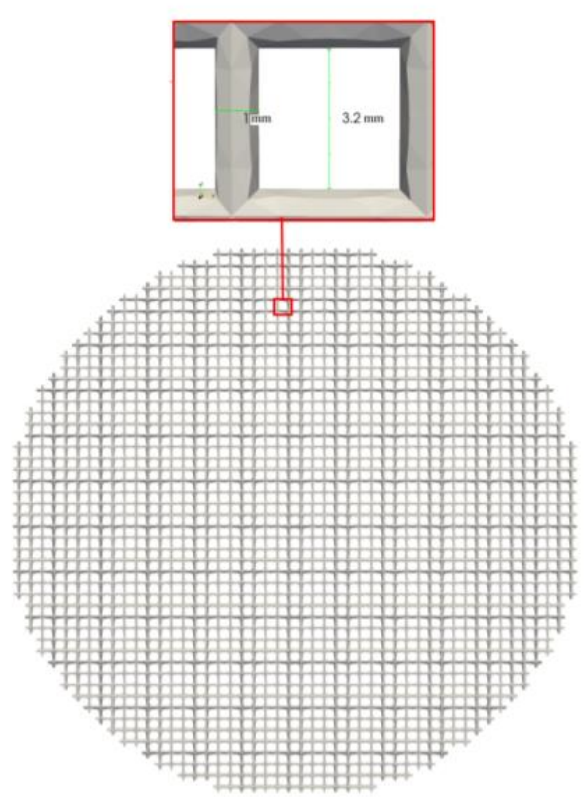

Fig. 1. Solid porous disk.

\section{NUMERICAL APPROACH}

These simulations are based on the BGK assumption and the regularized lattice Boltzmann technique that is applied to increase the stability and accuracy of the method. Turbulence is modelled using the static Smagorinky model and a grid refinement technique is applied.

\subsection{The Regularized Lattice Boltzmann equations}

The lattice Boltzmann (LB) equations write as (Nathen et al. 2018):

$$
f_{i}\left(x+c_{i} \Delta t, t+\Delta t\right)-f_{i}(x, t)=\Omega_{i}
$$

for $[i=1 \cdots N]$, where $f_{i}(x, t)$ is the probability to have a set of particles at location $x$ and time $t, c_{i}$ are the discrete lattice velocities, $N$ is the number of discrete velocities and $\Omega_{i}$ is the collision operator. The modelling of the collision term depends on the particular scheme BGK, MRT or RLB (Nathen et al. 2018). The macroscopic variables such as the fluid density $(\rho)$ and the momentum $(\rho u)$ are computed in terms of the particle distribution functions $f_{i}$, as:

$$
\rho=\sum_{i=1}^{N} f_{i} \quad \rho u=\sum_{i=1}^{N} f_{i} c_{i}
$$

The velocity distribution functions are expanded in a discrete Taylor series:

$$
f_{i}=f_{i}^{(0)}+\grave{\mathrm{o}} f_{i}^{(1)}+\grave{\mathrm{o}}^{2} f_{i}^{(2)}+\cdots
$$

where $\varepsilon$ is the Knudsen number. When the BGK assumption is made, $\Omega_{i}$ is expressed in term of $f_{i}$ and an equilibrium state $f_{i}^{e q}$ as:

$\Omega_{i}=-\frac{1}{\tau_{0}}\left(f_{i}-f_{i}^{e q}\right)$

where $\tau_{0}$ is the molecular relaxation time and $f_{i}^{e q}$ writes:

$f_{i}^{e q}=\rho \omega_{i}\left[1+\frac{c_{i} u}{c_{s}^{2}}+\frac{1}{2 c_{s}^{4}}\left(u u-c_{s}^{2} \delta\right) u u\right]$

$\omega_{i}$ are constant weights, $c_{s}$ is the lattice speed of sound and $\delta$ is the Kronecker delta.

In Regularized Lattice Boltzmann (RLB) method, an approximation of the first-order multi-scale expansion term is introduced (Nathen et al. (2018)):

$f_{i}^{n e q} \approx{\overline{f_{i}}}^{(1)}=-\frac{\Delta t}{\omega c_{s}^{2}} \omega_{i} Q_{i}: \nabla \rho u$

where, $Q_{i}$ is the first-order non equilibrium moment:

$Q_{i}=\sum c_{i} c_{i} f_{i}^{n e q}$

The non-equilibrium distribution function $f_{i}^{\text {neq }}$ is used to approximate the first-order multiscale expansion term in Eq. 3. This term is included in the BGK model, such that the regularized BGK algorithm writes:

$f_{i}\left(x+c_{i} \Delta t, t+\Delta t\right)-f_{i}(x, t)=(1-\omega) f_{i}^{(1)}(x, t)(8)$

\subsection{Turbulence Model}

Turbulence is modeled using the static LES Smagorinski model where only the largest scale are simulated while the smallest one are filtered. The unresolved scales are modeled by an effective relaxation time

$\tau_{\text {eff }}=\tau_{0}+\tau_{T}$

where $\tau_{0}$ and $\tau_{T}$ are the molecular and turbulent relaxation time, respectively (Yu et al. 2005). The corresponding effective viscosity is

$v_{e f f}=v_{0}+v_{T}$

where $v_{0}$ is the molecular viscosity defined as :

$v_{0}=\frac{1}{3}\left(\tau_{0}-\frac{1}{2}\right) c^{2} \Delta t$

and $v_{T}$ is the turbulent viscosity: 
D. Hamane et al. / JAFM, Vol. 14, No. 5, pp. 1521-1533, 2021.

$v_{T}=\frac{1}{3} \tau_{T} c^{2} \Delta t$

In the Smagorinsky model, the turbulent viscosity correction $v_{T}$ is given by the relationship (Nathen et al. 2013):

$v_{T}=\left(C_{s} \Delta x\right)^{2} \cdot|\Pi|$

where $C_{s}$ is the Smagorinsky constant, and $|\Pi|=\sqrt{\Pi_{i j} \Pi_{i j}}$ is the second-order moment of the nonequilibrium term of the distribution functions. According to (Yu et al. 2005) the turbulent relaxation time is expressed as :

$$
\tau_{T}=\frac{1}{2} \sqrt{\tau_{0}^{2}+\left(4 \sqrt{2}\left(C_{s} \Delta x\right)^{2} \frac{\tau_{\text {eff }}}{c_{s} \Delta t}|\Pi|\right)}-\tau_{0}
$$

There is no specific indication for $C_{s}$ value. In the literature, it is generally noted that the Smagorinsky's constant varies between 0.1 and 0.4.

\subsection{Computational Domain and Grid}

All computations are carried out on a threedimensional rectangular domain $(O, x, y, z)$. The freestream flow is aligned with the $(x)$ direction, $(y)$ is the horizontal crosswise direction and $(z)$ is the vertical crosswise one. The reference frame is centered on the center of the porous disk. The domain dimensions in the $(x),(y)$ and $(z)$ directions are, respectively, $L_{X}=25 D, L_{Y}=5 D$ and $L_{Z}=5 D$. Upstream and downstream boundaries are located respectively at $-5 D$ and $+20 D$ from the disk. Lateral boundaries are located at about $\pm 2.5 \mathrm{D}$ from the disk(Fig. 2).

Immersed boundary condition is applied on a solid porous disk and grid refinement techniques is used for the generation of Cartesian grid level. To select the optimal lattice size, test computations were performed on grids with different refinement levels. The mesh used for the computations is composed of three uniform cartesian mesh level (Fig. 3). The grid size is reduced from $\Delta x=D / 12.5$ far from the porous disk to $\Delta x=D / 50$ around the porous disk and its wake. The total number of mesh nodes is about $3.6 \cdot 10^{6}$.

\subsection{Boundary Conditions}

According to Latt and Chopard (2008), to improve the accuracy of the solution, the non local regularized boundary condition or Skordos model (Skordos (1993)) is recommended.

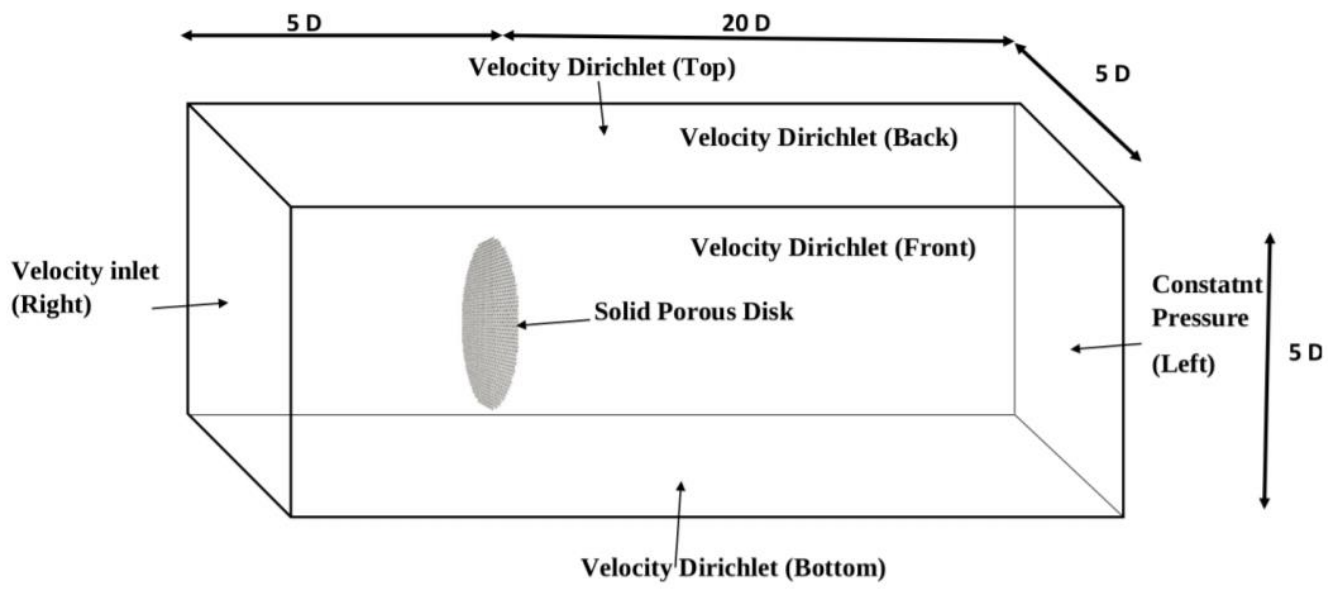

Fig. 2. View of the computational domain.

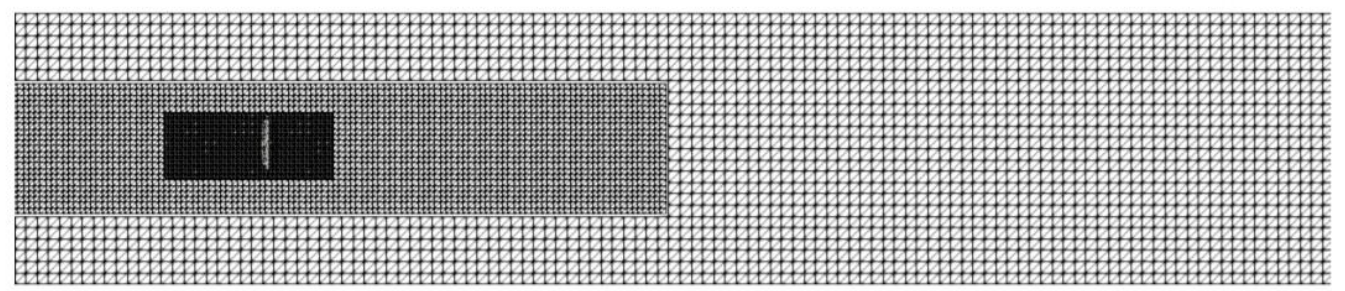

Fig. 3. View of the three level mesh refinement. 
This approach is thus applied and the following boundary conditions are set for external walls:

- Inlet Velocity condition is applied at Right boundary setting the Reynolds number $R e$, the free stream velocity $U_{0}$, the lattice velocity $U_{L B}$ and the fluid density $\rho=1.225 \mathrm{~kg} / \mathrm{m}^{3}$.

- Constant pressure is set at Left boundary.

- Velocity Dirichlet condition is set on lateral boundaries.

A sponge layer inside the computational domain is defined at the outlet boundary to avoid unphysical acoustic reflections (Wissocq et al. 2017).

For the solid porous disk, the conditions of Filippova and Hanel (1998) and the second-order interpolation scheme of Bouzidi et al. (2001) are compared.

\section{RESUlts}

For these simulations, computing facilities available on the Algerian research network (ARN DZGrid) were used. Each calculation took 18 hours on 72 processors. All computations are performed with the following parameters: the characteristic length that define the Reynolds number is fixed to $L_{r e f}=D$, the lattice velocity is set $U_{L B}=0.075$ and the fluid density is $\rho=1.225 \mathrm{~kg} / \mathrm{m}^{3}$.

Investigations are performed for different modeling parameters as the lattice velocity scheme, the value of the Smagorinsky's constant and the type of boundary condition on the solid porous disk.

\subsection{Velocity Contours}

The computations are first perform with the application of Bouzidi et al. (2001) boundary contition on the solid porous disk.

Figures 4 and 5 show, the obtained velocity contours for the lattice velocity schemes D3Q19 and D3Q27 respectively, $B C 1$ indicating Bouzidi et al. (2001) condition. These results are depicted for different values of the Smagorinsky's constant, in areas around the porous disk in the plane $(x z)$.

All velocity contours show similar flow patterns with recirculating flows in the near wake of the disk surface that vanish at about a distance of one diameter from the disk, to give place to one central recirculating flow around the porous disk axis.

However, the width of the circulatory flow and the maximal velocities computed with the different $C_{s}$ values and the lattice velocity schemes are slightly different. The results show that the width of the circulatory flow increases when the $C_{s}$ value increases while the maximal velocities slightly decrease. As for the influence of the lattice velocity schemes, it is found that the maximal velocities computed with both schemes are similar however the wakes are slightly larger in width and shorter in length with the D3Q27 scheme.

The flow patterns are similar to those obtained with classical CFD techniques applied for the simulation of wind turbine wakes modelled by a solid porous disk (see for e.g Ranjbar et al. (2020)).

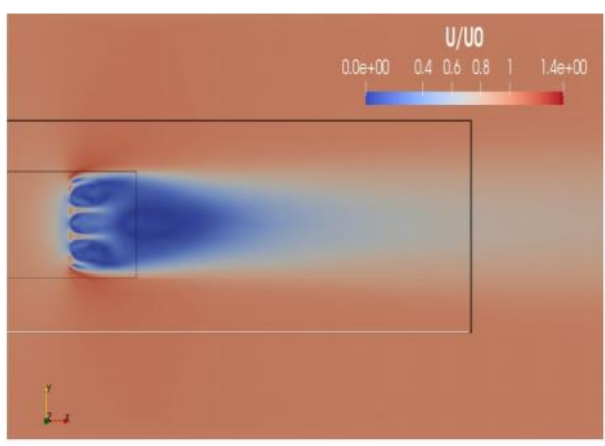

(a) $C_{s}=0.14$

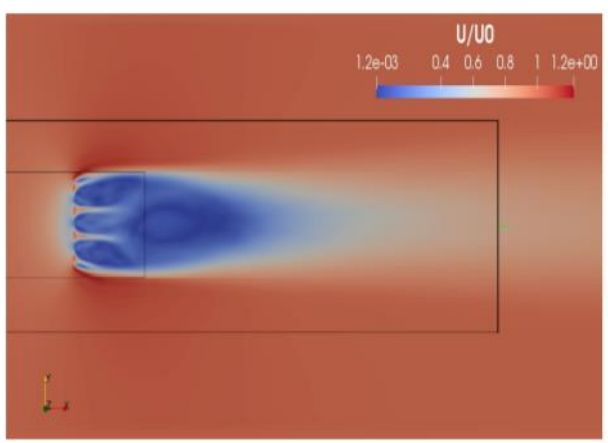

(b) $C_{s}=0.20$

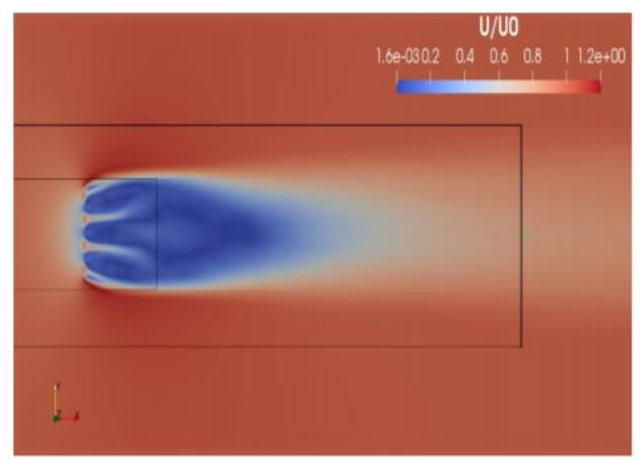

(c) $C_{s}=0.28$

Fig. 4. Contours of the normalized velocity magnitude around the disk in the plane $(x z)$, obtained with $B C 1$ condition and D3Q19 scheme.

\subsection{Contours of Vorticity}

The contours of the vorticity magnitude depicted in Figs. 6 and 7 show the influence of the value of the Smagorinski's constant value for D3Q19 and D3Q27 schemes, respectively. It is seen that the vorticity magnitude decreases as the $C_{s}$ value increases and that lower values are found with the D3Q27 scheme. As noticed in (Porte-Agel et al. 2020), the figures show that the wake grow in vertical direction as it moves downstream.

Figures 8 and 9, that depict the contours of the Zvorticity component, show the detachment of two 
D. Hamane et al. / JAFM, Vol. 14, No. 5, pp. 1521-1533, 2021.

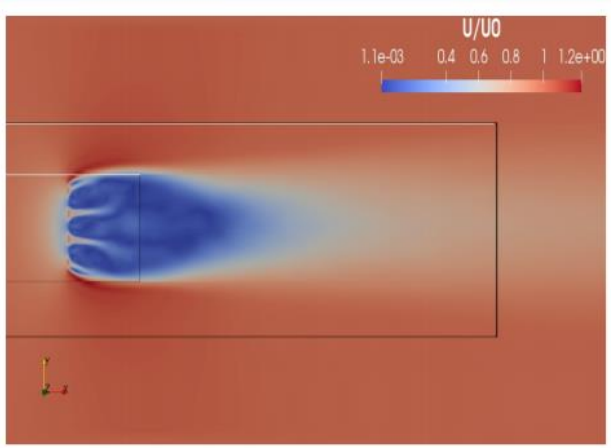

(a) $C_{s}=0.14$

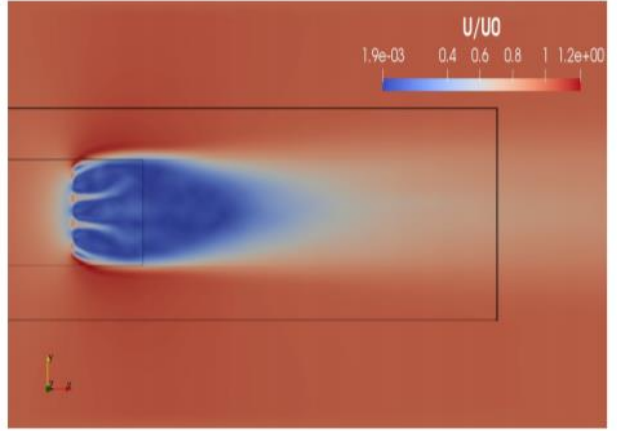

(b) $C_{s}=0.20$

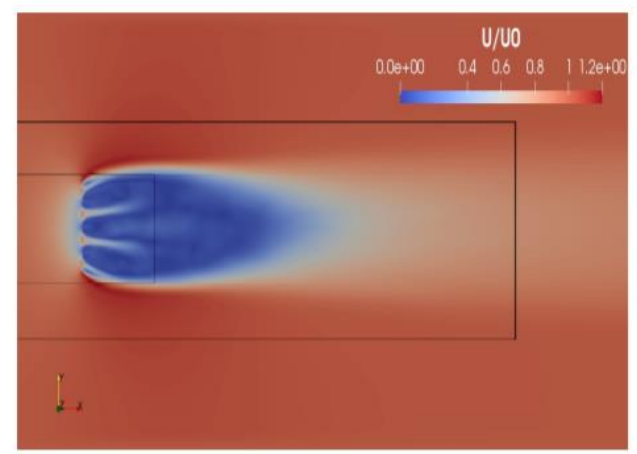

(c) $C_{s}=0.28$

Fig. 5. Contours of the normalized velocity magnitude around the disk in the plane $(x z)$, obtained with $B C 1$ condition and D3Q27 scheme.

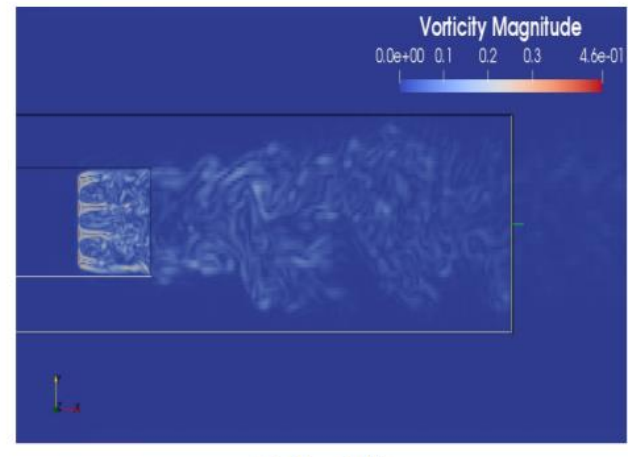

(a) $C_{s}=0.14$

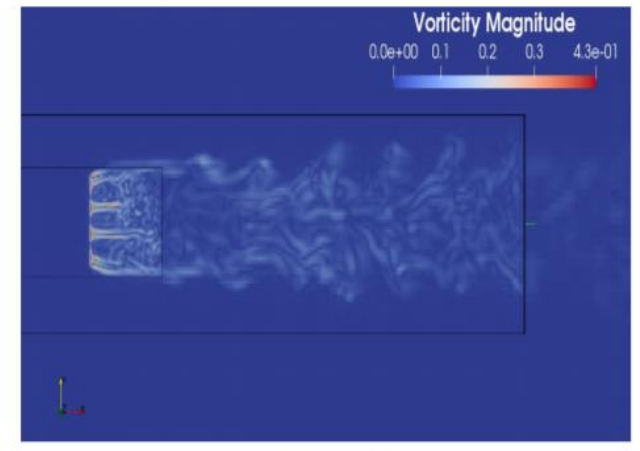

(b) $C_{s}=0.20$

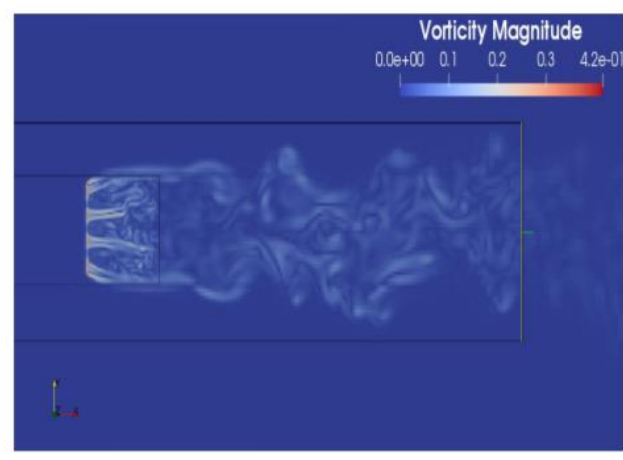

(c) $C_{s}=0.28$

Fig. 6. Contours of the normalized vorticity magnitude around the disk in the plane $(x z)$, obtained with $B C 1$ condition and D3Q19 scheme. 
D. Hamane et al. / JAFM, Vol. 14, No. 5, pp. 1521-1533, 2021.

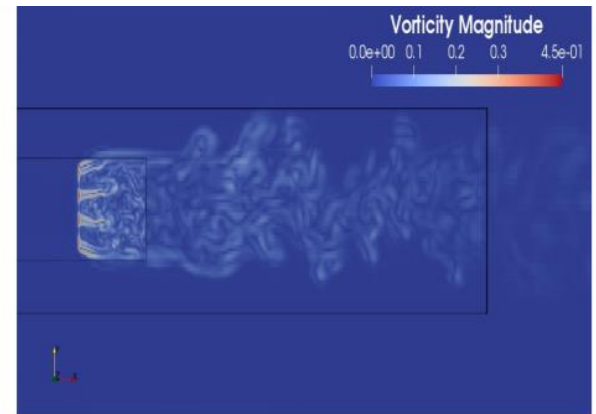

(a) $C_{s}=0.14$

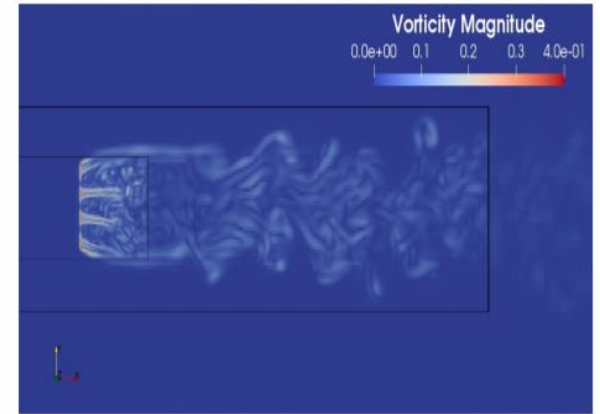

(b) $C_{s}=0.20$

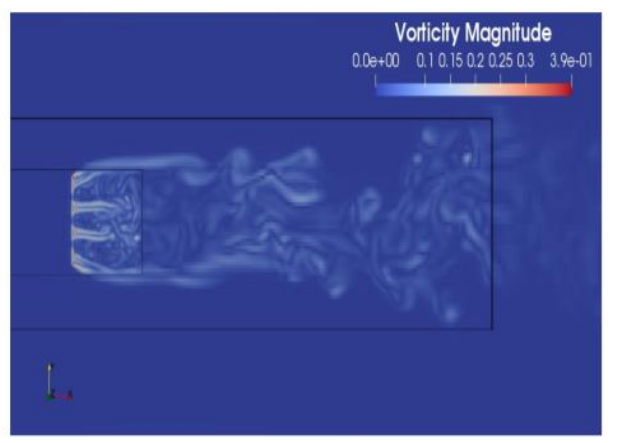

(c) $C_{s}=0.28$

Fig. 7. Contours of the normalized vorticity magnitude around the disk in the plane $(x z)$, obtained with $B C 1$ condition and D3Q27 scheme.

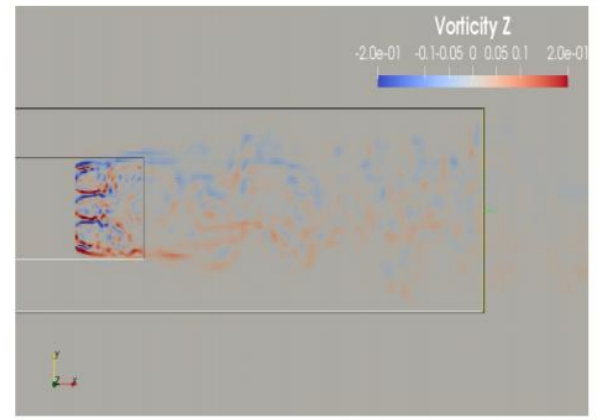

(a) $C_{s}=0.14$

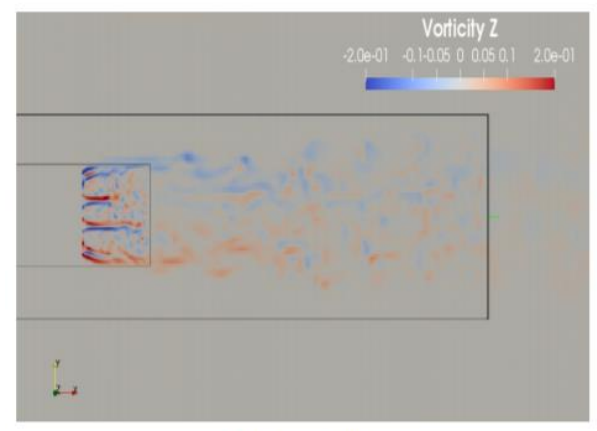

(b) $C_{s}=0.20$

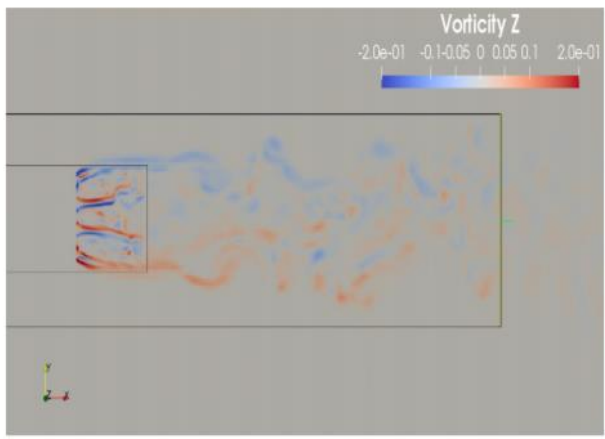

(c) $C_{s}=0.28$

Fig. 8. Contours of the normalized z-vorticity component around the disk in the plane $(x z)$, obtained with $B C 1$ condition and $D 3 Q 19$ scheme. 


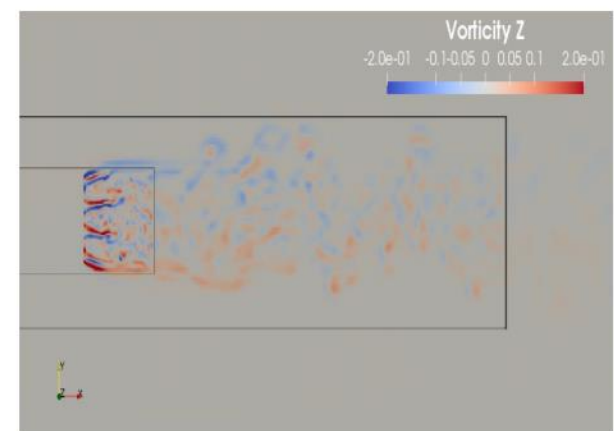

(a) $C_{s}=0.14$

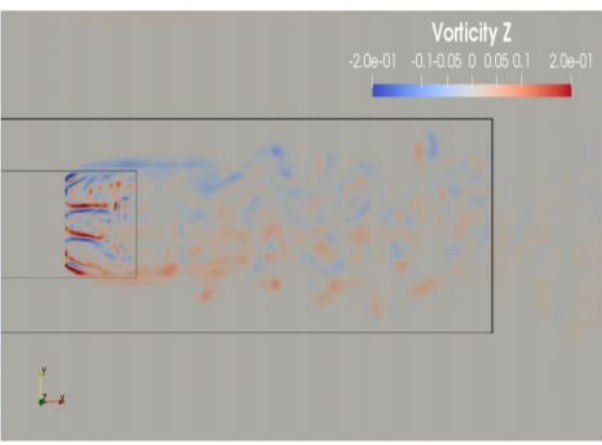

(b) $C_{s}=0.20$

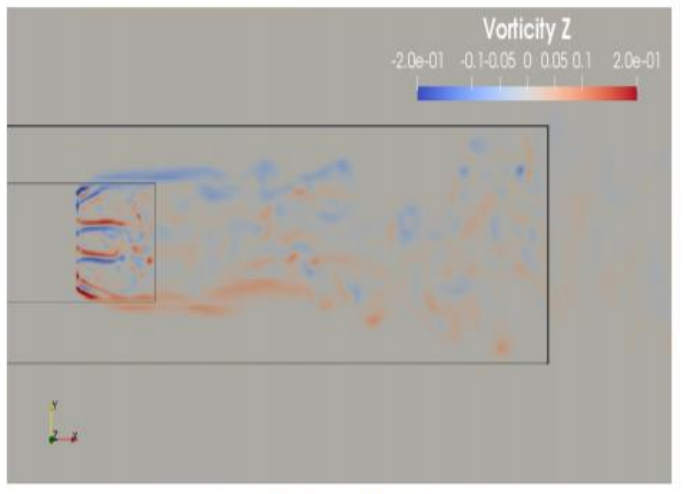

(c) $C_{s}=0.28$

Fig. 9. Contours of the normalized z-vorticity component around the disk in the plane $(x z)$, obtained with $B C 1$ condition and D3Q27 scheme.

contra-rotating vortices from the upper and lower bounds of the disk which propagate in the wake in the form of two longitudinal vortices. Smaller eddies detach from the disk meshes, but they disappear in the near wake.

\subsection{Influence of Solid Boundary Condition}

To study the influence of the boundary condition to apply on the solid porous disk, computations are also performed with Filippova and Hanel (1998) condition. The results obtained with D3Q27 scheme and the different Smagorinsky's constant values are depicted in Figs. 10 to 12. As previously, the velocity contours show that the width of the circulatory flow increases when the $C_{s}$ value increase while the maximal velocities decrease, but the wake width and lenght are different to those obtained with Bouzidi et al. (2001) condition. The zcomponent vorticity contours show also two contrarotating vortices that detach from the upper and lower bounds and smaller eddies that detach from the disk surface.

\subsection{Velocity Profiles}

For a better analysis of the differences obtained with the parameters investigated in this study, the velocity profiles are compared to experimental data provided by S. Aubrun that are published in Sumner et al. (2013), and to the analytical model of Bastankhah and Porte-Agel (2014) which writes as:

$$
\begin{aligned}
\frac{\Delta u}{U_{0}}=\left(1-\sqrt{1-\frac{C_{T}}{8(\sigma / D)^{2}}}\right) \\
\exp \left(-\left[\frac{y^{2}}{2 \sigma^{2}}+\frac{\left(z-z_{h}\right)^{2}}{2 \sigma^{2}}\right]\right)
\end{aligned}
$$

Where

$\frac{\sigma}{D}=k \frac{x}{D}+v$

$v$ is the initial wake width, equal to $0.2 \sqrt{\beta}$ and

$\beta=\frac{1+\sqrt{1-C_{T}}}{2 \sqrt{1-C_{T}}}$

The obtained results are depicted in Fig. 13 to 15 , at different locations in the porous disk wake.

As this was reported in other studies of wind turbine wakes in uniform inflow (Porte-Agel et al. 2020), the figures show that the streamwise velocity profiles have an axisymmetric Gaussian distribution in the region of far wake with a slight disagreement between the velocity-deficit profiles and the Gaussian distribution at the wake edges.

The figures show also that the different velocity profiles are in good agreement with experimental data, mainly with $C_{s}=0.14$ and 0.20 however, the 
D. Hamane et al. / JAFM, Vol. 14, No. 5, pp. 1521-1533, 2021.

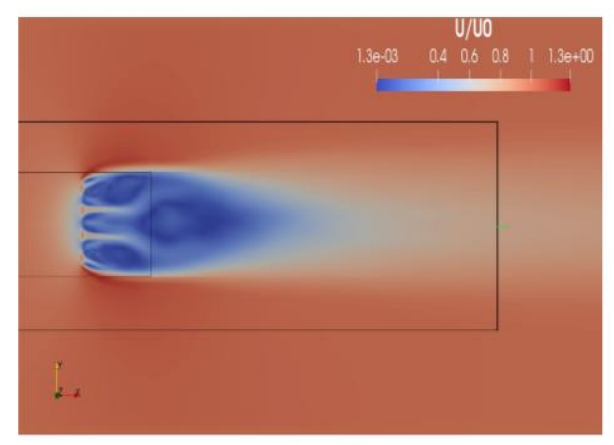

(a) $C_{s}=0.14$

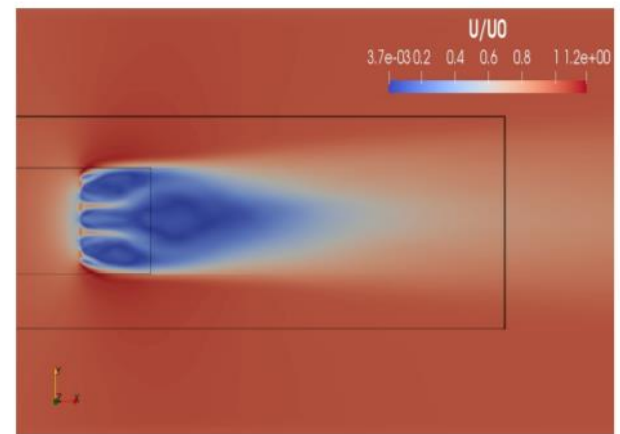

(b) $C_{s}=0.20$

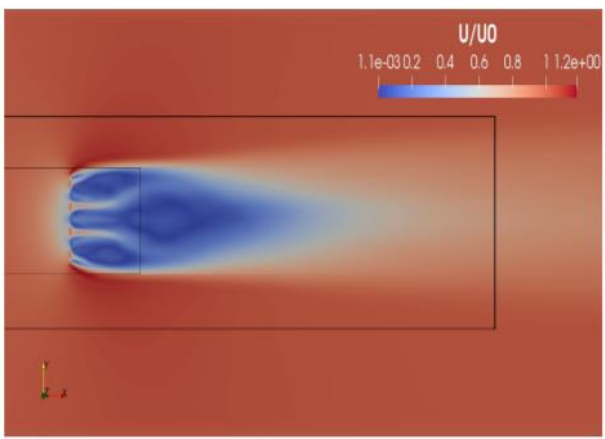

(c) $C_{s}=0.28$

Fig. 10. Contours of the normalized velocity magnitude around the disk, in the plane $(x z)$, obtained with $B C 2$ condition and $\mathrm{D3Q} 27$ scheme.

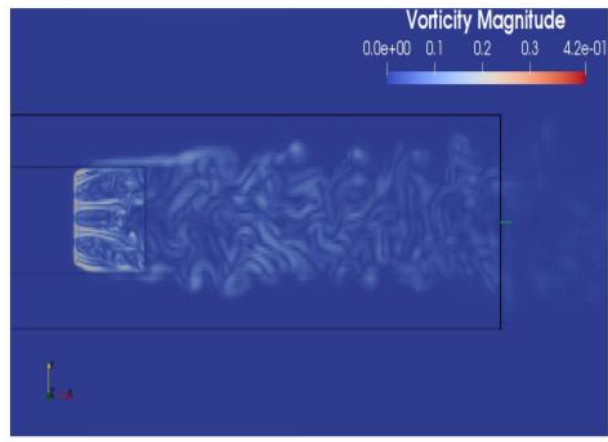

(a) $C_{s}=0.14$

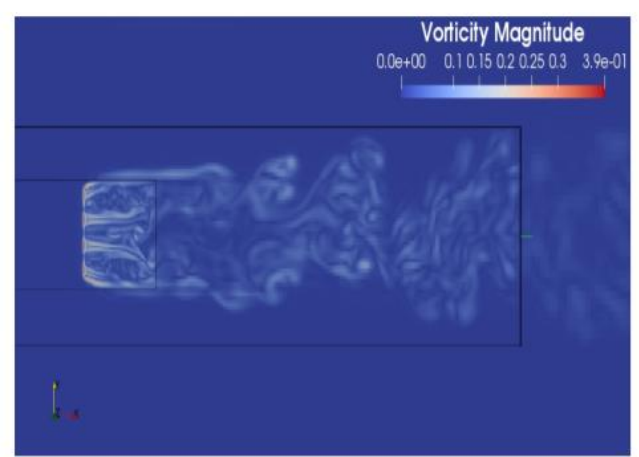

(b) $C_{s}=0.20$

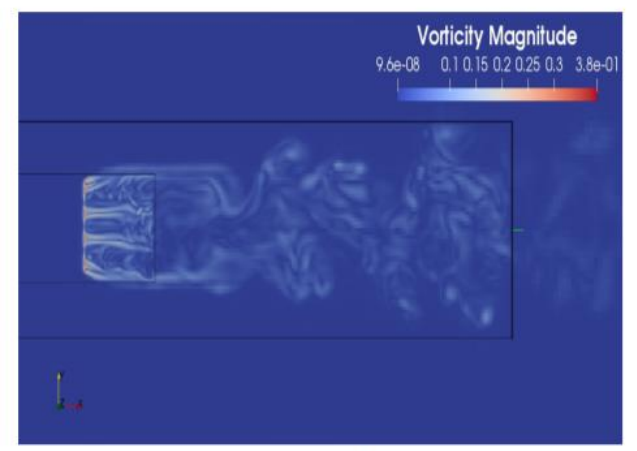

(c) $C_{s}=0.28$

Fig. 11. Contours of the normalized vorticity magnitude around the disk, in the plane $(x z)$, obtained with $B C 2$ condition and $\mathrm{D} 3 \mathrm{Q} 27$ scheme. 
D. Hamane et al. / JAFM, Vol. 14, No. 5, pp. 1521-1533, 2021.

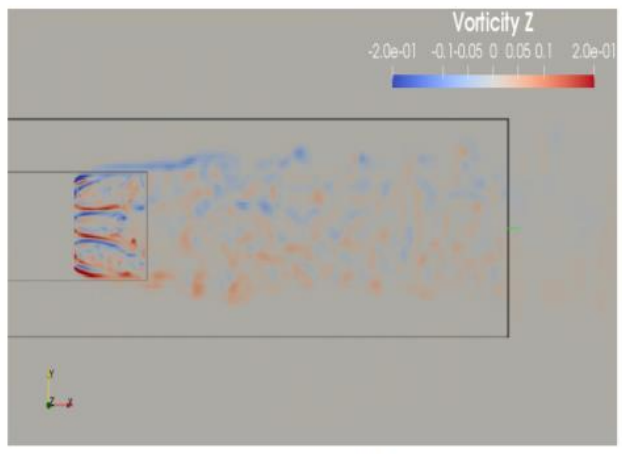

(a) $C_{s}=0.14$

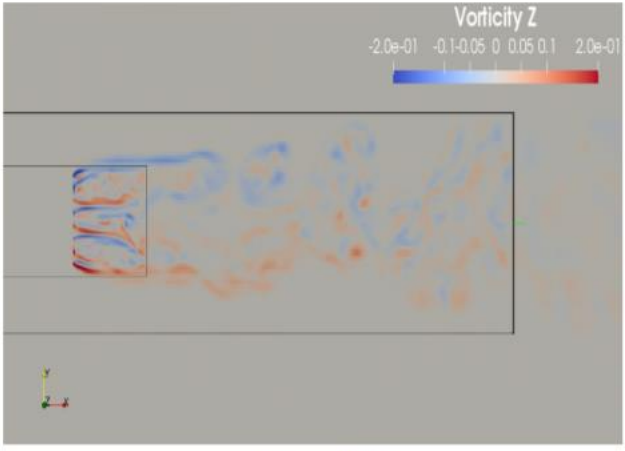

(b) $C_{s}=0.20$

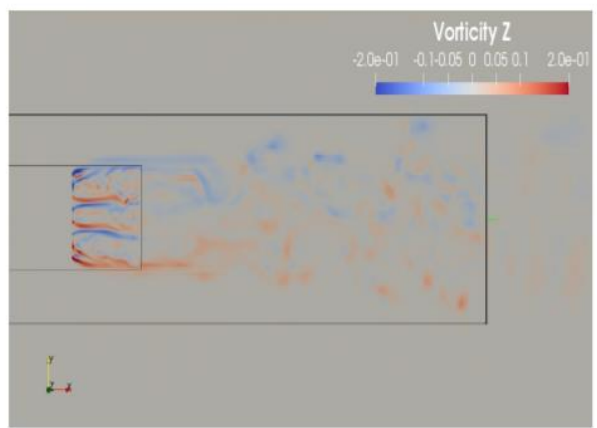

(c) $C_{s}=0.28$

Fig. 12. Contours of the normalized z-vorticity component around the disk, in the plane $(x z)$, obtained with $B C 2$ condition and D3Q27 scheme.

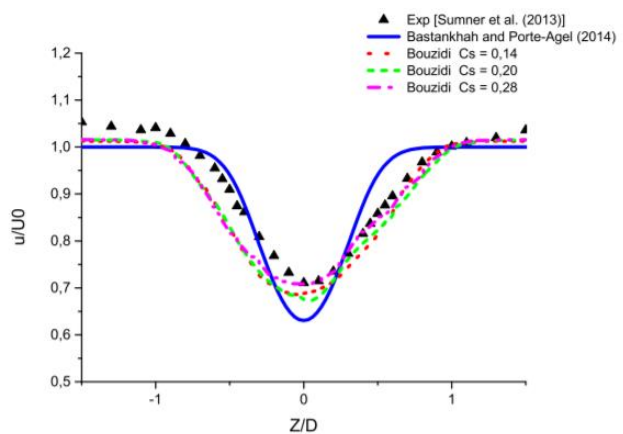

(a) BC1 - D3Q19 scheme

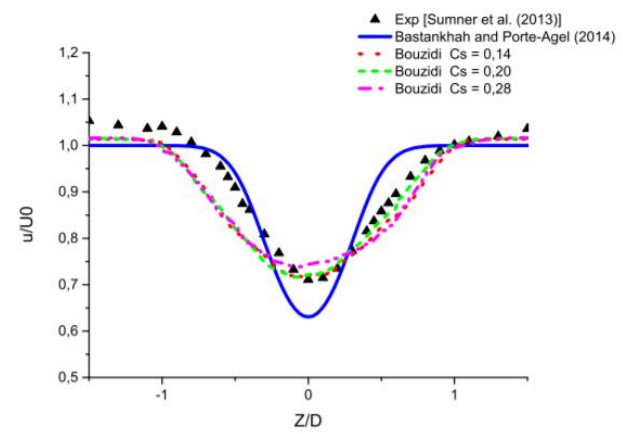

(b) BC1 - D3Q27 scheme

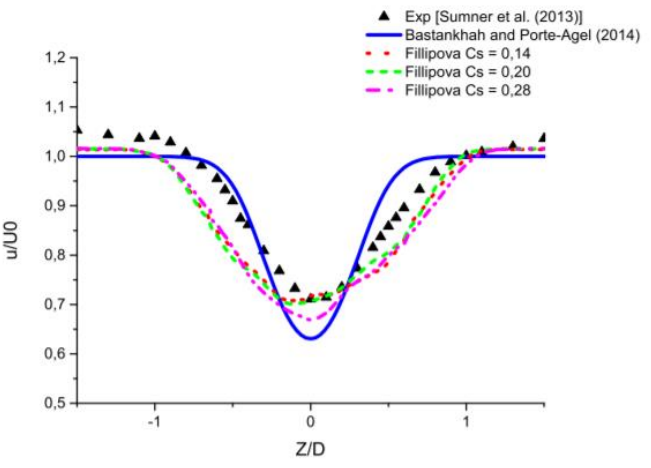

(c) BC2 - D3Q27 scheme

Fig. 13. Vertical velocity profiles of $u / U_{0}$ component, at $x=6 D$ downstream the dis. 
D. Hamane et al. / JAFM, Vol. 14, No. 5, pp. 1521-1533, 2021.

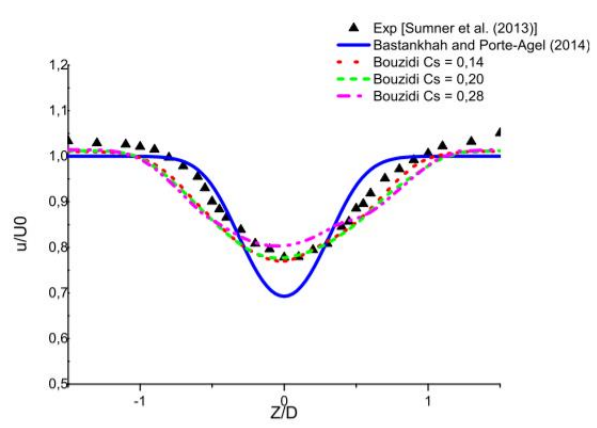

(a) BC1 - D3Q19 scheme

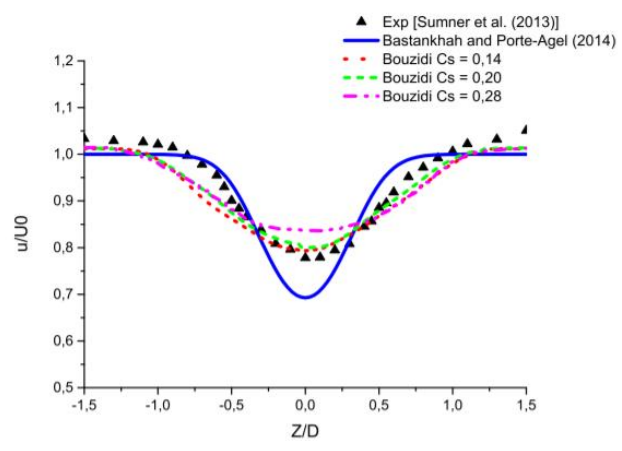

(b) $\mathrm{BC} 1$ - D3Q27 scheme

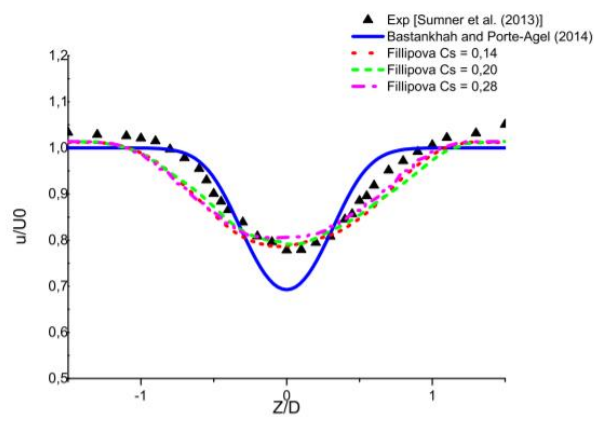

(c) BC2 - D3Q27 scheme

Fig. 14. Vertical velocity profiles of $u / U_{0}$ component, at $x=8 D$.

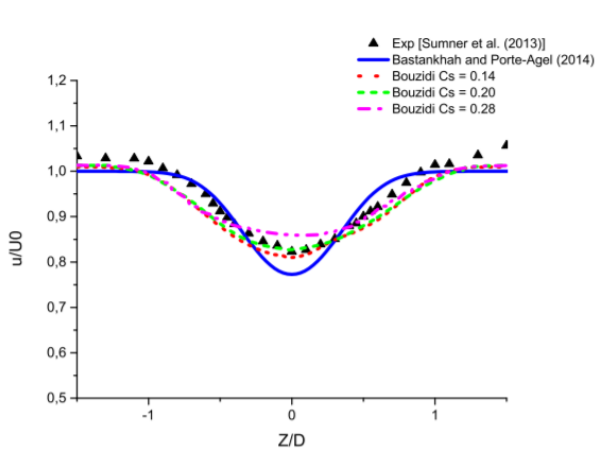

(a) BC1 - D3Q19 scheme

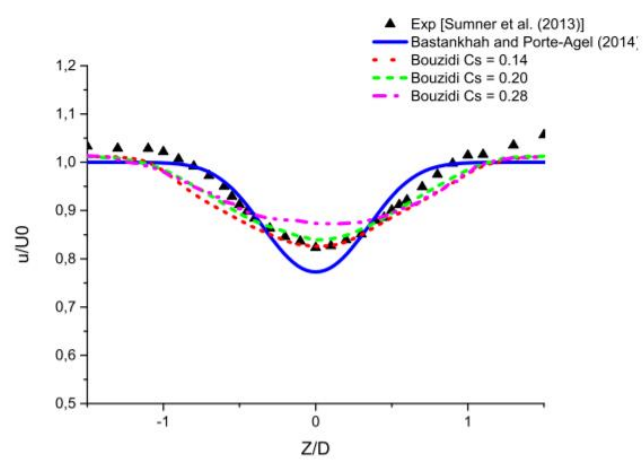

(b) BC1 - D3Q27 scheme

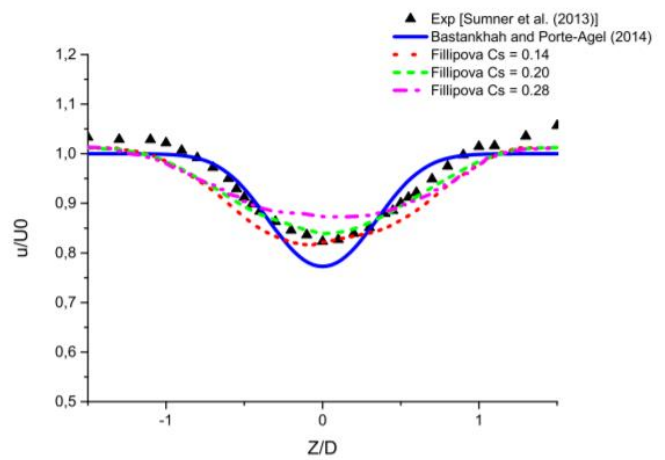

(c) BC2 - D3Q27 scheme

Fig. 15. Vertical velocity profiles of $u / U_{0}$ component, at $x=10 D$. 
computed wake is larger. Moreover, the velocity deficit is better reproduced, compared to the analytical model.

The horizontal velocity profiles in the vicinity of the disk are used to compute the induction factor. The obtained values are given in Tables 1 and 2 for both types of boundary condition respectively. The errors are calculated with respect to data given by (Espana 2009). It is seen that lower errors are found with (Bouzidi et al. 2001) condition and with $C_{s}=0.20$.

Table 1 Induction factor - BC1 condition.

\begin{tabular}{|c|c|c|c|c|}
\hline Schemes & \multicolumn{2}{|c|}{ D3Q19 } & \multicolumn{2}{c|}{ D3Q27 } \\
\hline Cs & a & $\begin{array}{c}\text { error } \\
(\%)\end{array}$ & $\mathrm{a}$ & $\begin{array}{c}\text { error } \\
(\%)\end{array}$ \\
\hline 0.14 & 0.188 & -3.59 & 0.199 & 2.05 \\
\hline 0.20 & 0.198 & +1.54 & 0.203 & 4.10 \\
\hline 0.28 & 0.198 & +1.54 & 0.208 & 6.67 \\
\hline
\end{tabular}

Table 2 Induction factor - BC2 condition.

\begin{tabular}{|c|c|c|c|c|}
\hline Schemes & \multicolumn{2}{|c|}{ D3Q19 } & \multicolumn{2}{c|}{ D3Q27 } \\
\hline Cs & a & $\begin{array}{c}\text { error } \\
(\%)\end{array}$ & $\mathrm{a}$ & $\begin{array}{c}\text { error } \\
(\%)\end{array}$ \\
\hline 0.14 & - & - & 0.183 & -6.15 \\
\hline 0.20 & 0.174 & -10.77 & 0.184 & -5.64 \\
\hline 0.28 & 0.179 & -8.21 & 0.179 & -8.21 \\
\hline
\end{tabular}

\section{SUMMARY AND CONCLUSION}

This study shows the ability of the LBM to reproduce the wind turbine wake. On the whole, these results show flow patterns similar to those obtained with others computations techniques applied for the simulation of wind turbine wakes. But the results vary somewhat with the type of boundary condition, the lattice velocity scheme and the $C_{s}$ value. In summary:

- The width of the circulatory flow increase when the $C_{s}$ value increases.

- The maximal velocities decrease when the $C_{s}$ value increases.

- The width of the circulatory flow computed using Filippova boundary condition (BC2) on a solid porous disk is larger than that get in Bouzidi boundary condition (BC1). - Slightly higher maximal velocities are found with Bouzidi boundary conditions (BC1).

As for the velocity profiles, the obtained results are in good agreement with experimental data, and lower errors are found for the induction factor with Bouzidi condition (BC1) and with $C_{s}=0.20$.

\section{ACKNOWLEDGMENTS}

The authors would like to thank Professor Sandrine Aubrun, actually at Ecole Centrale de Nantes (France), for kindly providing its experimental data. Computational resources have been provided by the research center for scientific and technical information Algiers, Algeria (CERIST, www.arn.dz).

\section{REFERENCES}

Ameur, K., C. Masson and P. J. Eecen (2011). 2d and $3 \mathrm{~d}$ numerical simulation of the windrotor/nacelle interaction in an atmospheric boundary layer. Journal of Wind Engineering and Industrial Aerodynamics 99, 833-844.

Aubrun, S., S. Loyer, P. Hancock and P. Hayden. (2013). Wind turbine wake properties: Comparison between a non-rotating simplified wind turbine model and a rotating model. Journal of Wind Engineering and Industrial Aerodynamics 120, 1-8.

Bastankhah, M. and F. Porte-Agel (2014). A new analytical model for wind-turbine wakes. Renewable Energy 70, 116-123.

Bouzidi, M., M. Firdaouss and P. Lallemand (2001). Momentum transfer of a boltzmannlattice fluid with boundaries. Physics of Fluids 13, 34523459 .

Chen, X. P. (2015). Applications of lattice boltzmann method to turbulent flow around twodimensional airfoil, engineering applications of computational fluid mechanics. Engineering Applications of Computational Fluid Mechanics 6(4), 572-580.

Englberger, A. and A. Dornbrack (2016). Impact of neutral boundary-layer turbulence on windturbine wakes: a numerical modelling study. Boundary-Layer Meteorology 162, 427-449.

Englberger, A. and A. Dornbrack (2017). Impact of the diurnal cycle of the atmospheric boundary layer on wind-turbine wakes: A numerical modelling study. Boundary-Layer Meteorology.

Espana, G. (2009). Etude expérimentale du sil- lage lointain deséoliennes á axe horizon- tales au moyen d'une modelisation simplifiéeen couche limite atmospherique. $\mathrm{Ph}$. D. thesis, Universite d'Orléans (France).

Espana, G., S. Aubrun and S. L. and P. Devinant (2012). Wind tunnel study of the wake meandering downstream of a modelled wind turbine as an effect of large scale turbulent eddies. Journal of Wind Engineering and Industrial Aerodynamics 101, 24- 33. 
Filippova, O. and D. Hanel (1998). Grid refinement for lattice-bgk models. Journal of Computational Physics 147, 219-228.

Gomez-Elvira, R., A. Crespo, E. Migoya, F. Manuel and J. Hernandez (2005). Anisotropy of turbulence in wind turbine wakes. Journal of Wind Engineering and Industrial Aerodynamics 93, 797-814.

Gourdain, N., T. Jardin, R. Serre, S., Prothin and J. Moschetta (2018). Application of a lattice boltzmann method to some challenges related to micro-air vehicles. International Journal of Micro Air Vehicles 10(3), 285- 299.

Grondeau, M., S. Guillou, P. Mercier and E. Poizot (2019). Wake of a ducted vertical axis tidal turbine in turbulent flows, $\mathrm{lbm}$ actuator-line approach. Energies 12(4273).

Jafari, Y., M. Taeibi-Rahni, M. Haghshenas and P. Ramian. (2018). Lattice boltzmann numerical investigation of inner cylindrical pin-fins configuration on nanofluid natural convective heat transfer in porous enclosure. Journal of Applied Fluid Mechanics 11(3), 801-816.

Kasmi, A. E. and C. Masson (2008). An extended $k-\varepsilon$ model for turbulent flow through horizontal-axis wind turbines. Journal of Wind Engineering and Industrial Aerodynamics 96, $103-122$.

Khazaeli, R., S. Mortazavi and M. Ashrafizaadeh (2015). Application of an immersed boundary treatment in simulation of natural convection problems with complex geometry via the lattice boltzmann method. Journal of Applied Fluid Mechanics 8(2), 309-321.

Kumar, G. V. S., D. A. Perumal and A. K. Dass (2010). Numerical simulation of viscous flow over a circular cylinder using lattice boltzmann method. In Proceedings of the 37th National \& 4th International Conference on Fluid Mechanics and Fluid Power, Number FMFP10CF-35.

Latt, J. and B. Chopard (2008). Straight velocity boundaries in the lattice boltzmann method. Physical Reviewe 77, 056703.

Latt, J., O. Malaspinas, D. Kontaxikas, A. Parmigiani, D. Lagrava, F. Brogi, M. B. Belgacem, Y. Thorimbert, S. Leclaire, S. Li, F. Marson, J. Lemus, C. Kotsalos, R. Conradin, C. Coreixas, R. Petkantchin, F. Raynaud, J. Beny and B. Chopard (2020). Palabos: Parallel lattice boltzmann solver. Computers and Mathematics with Applications.

Liu, T., G. Liu, Y. Ge, H. Wu and W. Wu (2008). Extended lattice boltzmann equation for simulation of flows around bluff bodies in high reynolds number. In $B B A A V I$ International Colloquium on: Bluff Bodies Aerodynamics \& Applications, Milano, Italy.
Nathen, P., D. Gaudlitz, J. Kratzke and M. J. Krause (2013, June). An extension of the latticeboltzmann method for simulating turbulent flows around rotating geometries of arbitrary shape. In F. Dynamics and C. located Conferences (Eds.), 21st AIAA Computational Fluid Dynamics Conference, San Diego, CA. American Institute of Aeronautics and Astronautics.

Nathen, P., D. Gaudlitz, M. J. Krause and N. A. Adams (2018). On the stability and accuracy of the bgk, mrt and rlb boltzmann schemes for the simulation of turbulent flows. Communication in Computational Physics 23(3), 846-876.

Porte-Agel, F., M. Bastankhah and S. Shamsoddin (2020). Wind-turbine and wind-farm flows: A review. Boundary-Layer Meteorology 174, 159.

Ranjbar, M. H., H. Z. Kia, S. A. Nasrazadani, K. Gharali and J. Nathwani (2020). Experimental and numerical investigations of actuator disks for wind turbines. Energy Science and Engineering, 1-16.

Rullaud, S., F. Blondel and M. Cathelain (2018). Actuator-line model in a lattice boltzmann framework for wind turbine simulations. Journal of Physics: Conference Series, IOP Publishing (1037), 022023. hal-01917527.

Sanderse, B., S. Pijl and B. Koren (2011). Review of computational fluid dynamics for wind turbine wake aerodynamics. Wind Energy 14(7), 799819.

Skordos, P. (1993). Initial and boundary conditions for the lattice boltzmann method. Physical Review E 48(6), 4823-4842.

Sumner, J., G. Espana, C. Masson and S. Aubrun (2013). Evaluation of rans/actuator disk modelling of wind turbine wake flow using wind tunnel measurements. International Journal of Engineering Systems Modelling and Simulation 5(1/2/3).

Wissocq, G., N. Gourdain, O. Malaspinas and A. Eyssartier (2017). Regularized characteristic boundary conditions for the latticeboltzmann methods at high reynolds number flows. Journal of Computational Physics 331, 1-18.

Wu, Y. T. and F. Porte-Agel (2011). Large-eddy simulation of wind-turbine wakes: Evaluation of turbine parametrisations. BoundaryLayer Meteorology 138, 345-366.

Yu, H., S. Sharath, Girimaji and L. S. Luo (2005). Dns and les of decaying isotropic turbulence with and without frame rotation using lattice boltzmann method. Journal of Computational Physics 209, 599-616. 